\title{
Peripheral Inflammatory Blood Markers in Diagnosis of Glioma and IDH Status
}

\author{
Gaurav Sharma ${ }^{1}$ Shashi Kant Jain ${ }^{1}$ Virendra Deo Sinha ${ }^{1}$ \\ 1Department of Neurosurgery, Sawai Man Singh Medical College \\ Jaipur, Jaipur, India \\ Address for correspondence Gaurav Sharma, MS, MCh, \\ Department of Neurosurgery, Sawai Man Singh Medical College, \\ Jaipur, India (e-mail:dr.gaurav_87@yahoo.co.in).
}

J Neurosci Rural Pract:2021;12:88-94

\begin{abstract}
Keywords

- inflammatory markers

- glioma

- IDH status

- oncology
\end{abstract}

Objective Gliomas are the most common intracranial tumors. Histopathology and neuroimaging are the main modalities used for diagnosis and treatment response monitoring. However, both are expensive and insensitive methods and can cause neurological deterioration. This study aimed to develop a minimally invasive peripheral inflammatory biomarker for diagnosis of glioma, its grade, and isocitrate dehydrogenase (IDH) status.

Materials and Methods Patients undergoing surgery for glioma, acoustic neuroma, and meningioma between January 2019 and December 2019 were included. Preoperative neutrophil/lymphocyte ratio (NLR), derived NLR (dNLR), platelet/lymphocyte ratio (PLR), lymphocyte/monocyte ratio (LMR), eosinophil/lymphocyte ratio (ELR), and prognostic nutritional index (PNI) were calculated. Histopathology and immunohistochemistry (IHC) staining were done postoperatively.

Results A total of 154 patients of glioma, 36 patients of acoustic neuroma, 58 patients of meningioma, and 107 healthy controls were included. dNLR showed the maximum area under the curve (AUC) (0.656639) for diagnosis of glioma from other tumors and among combinations. dNLR +NLR showed the maximum AUC (0.647865). Maximum AUC for glioblastoma multiforme (GBM) versus other grades and among combinations was shown by NLR (0.83926). NLR + dNLR had the maximum AUC (0.764794). NLR showed significant $p$ value in differentiating IDH wild from IDH mutant GBM.

Conclusion dNLR has the maximum diagnostic value in diagnosing glioma from other tumors. NLR (AUC $=0.83926$ ) showed the highest accuracy for GBM diagnosis and may be a parameter in predicting the grade of glioma; also, it has maximum diagnostic value in differentiating IDH wild GBM from IDH mutant GBM. These peripheral inflammatory parameters may prove to be sensitive and cost-effective markers for glioma diagnosis, predicting grade of glioma, monitoring of treatment response, and in predicting recurrence.

\section{Introduction}

Glioma, the commonest intracranial tumor accounts for approximately $81 \%$ of malignant tumors of brain. ${ }^{1}$ The most frequent type is glioma grade IV (glioblastoma multiforme [GBM]), with incidence of approximately 3.5/100,000 per year. ${ }^{2}$ Despite the advancements made in the treatment of glioma, the 5-year survival rate remains poor. ${ }^{3}$ At present definitive diagnosis is made by histopathological studies of tumor tissue obtained by either resection or biopsy, whereas neuroimaging (CT or MRI) is used for assessment of treatment response, staging, and recurrence. ${ }^{4}$ However, imaging and histopathological tests are expensive and results in
DOI https://doi.org/ 10.1055/s-0040-1721166 ISSN 0976-3147.
(C) 2021. Association for Helping Neurosurgical Sick People.

This is an open access article published by Thieme under the terms of the Creative Commons Attribution-NonDerivative-NonCommercial-License, permitting copying and reproduction so long as the original work is given appropriate credit. Contents may not be used for commercial purposes, or adapted, remixed, transformed or built upon. (https://creativecommons.org/licenses/by-nc-nd/4.0/)

Thieme Medical and Scientific Publishers Pvt. Ltd. A-12, 2nd Floor, Sector 2, Noida-201301 UP, India 
neurological injury. ${ }^{5}$ Moreover, glioma has no serum marker that can be applied for its diagnosis, staging, monitoring of response to treatment modalities, and recurrence. So, there is need of developing sensitive but cost-effective markers for glioma, which can predict its diagnosis preoperatively and also help in monitoring of treatment response and recurrence.

Earlier studies have highlighted the involvement of inflammatory process in pathogenesis of many solid cancers, and inflammation severity may be correlated with the grade of tumor. ${ }^{6}$ Inflammation results in changes in levels of local and circulating neutrophils, lymphocytes, monocytes, eosinophils, and albumin. These serum markers are easy to measure and are low cost. Changes in the level of these markers and their ratios like neutrophil/lymphocyte (NLR), lymphocyte/monocyte (LMR) and platelet/lymphocyte (PLR) have been used as markers for host inflammation in many solid tumors, including prostate cancer, esophageal cancer, hepatic cancer, colon cancer, and lung cancer. These markers have aided in patient stratification to treatment and also help in predicting survival of these patients. ${ }^{7-10}$ Increased preoperative NLR has been suggested as a marker of poor survival in patients with GBM in earlier studies. ${ }^{11,12}$ Unfortunately, there are only limited studies focusing on the diagnostic value of such inflammatory parameters in glioma. Despite many studies suggesting the antitumor effect of eosinophils in solid tumors like colon, ${ }^{13}$ bladder, and lung cancer, ${ }^{14}$ there is scarcely any study focusing on role of eosinophils in glioma. We also included eosinophils in our study and assessed their role as inflammatory markers in patients with glioma.

Studies have also suggested an association of these inflammatory markers with the molecular status of glioma, ${ }^{15}$ and NLR has been suggested as the single best marker to differentiate isocitrate dehydrogenase (IDH)-mutated and wild type GBM.

The aim of the present study is to diagnose glioma, and determine its grade and IDH status preoperatively, using these peripheral inflammatory parameters.

\section{Materials and Methods}

\section{Study Population}

Patients with glioma, acoustic neuroma, and meningioma who underwent surgery between January 2019 and December 2019 in our institution were included. Following are the inclusion criteria: (1) Tumour diagnosis, grading verified histologically in resected specimen. (2) No preoperative radiotherapy, chemotherapy, or any cancer specific treatment. (3) No conditions that can alter the levels of blood parameters in study such as infections, renal or hepatic dysfunction. (4) Informed consent.

One fifty-four patients of glioma, 58 patients of meningioma, 36 patients of acoustic neuroma, and 107 healthy controls were included in the study.

\section{Data Collection}

Blood samples were taken within 3 days prior to surgery. Total and differential leucocyte counts, platelet counts, and serum albumin were done. Preoperative derived NLR
$(\mathrm{dNLR}=\mathrm{ANC} /[\mathrm{WBC}-\mathrm{ANC}]), \mathrm{PLR}, \mathrm{LMR}$, eosinophil/lymphocyte (ELR)and prognosticnutritionalindex $(\mathrm{PNI}=[10 \times$ serumalbumin $(\mathrm{g} / \mathrm{dL})]+[0.005 \times$ lymphocytes/L]) were calculated. Age, sex, histological diagnosis, grade of tumor, and molecular subtype were collected. mmunohistochemistry (IHC) staining was done to determine IDH status in patients with glioma.

\section{Statistical Analysis}

The association of inflammatory markers with tumor grade was determined using the Kruskal-Wallis test. Correlation was judged by the Pearson correlation test. The diagnostic performance of these markers was evaluated by calculating the area under the curve obtained from receiver operating characteristic (ROC) curve. $p$ value $<0.05$ was taken as statistically significant.

\section{Results}

\section{Demographics}

One fifty-four patients of glioma, 58 patients of meningioma, 36 patients of acoustic neuroma, and 107 healthy controls were included in present study. Demographic details of the patients are shown in - Table $\mathbf{1}$. The median age (years) for patients with acoustic neuroma, meningioma, glioma, and healthy controls were 49.5, 54.5, 42 and 41, respectively. Glioma group consisted of 86 (55.84\%) males and $68(44.16 \%)$ females. Glioma patients were further classified as grade I in $26(16.84 \%)$, grade II in $38(24.67 \%)$, grade III in $32(20.78 \%)$, and grade IV in $58(37.66 \%)$ patients. The IDH mutation rate in patients with glioma was $31.82 \%$ (49/154).

\section{Comparison of Preoperative Inflammatory Markers among Various Study Groups}

As shown in - Table 1, total leucocyte count (TLC), neutrophils, and NLR were significantly increased ( $p$ value $<0.05$ ) in patients with glioma in comparison to acoustic neuroma, meningioma, and healthy controls. A significant increase ( $p$ value $<0.05)$ in eosinophil count was observed in glioma group than other study groups. A significant decrease ( $p$ value $<0.05$ ) in lymphocyte count was observed in glioma group than meningioma and healthy controls. PNI was significantly low $(p$ value $<0.05$ ) in glioma patients than other groups, except for patients with meningioma. LMR in glioma patients was significantly lower $(p$ value $<0.05)$ than other groups, except in meningioma group.

\section{Inflammatory Markers and Glioma Grade}

Patients with glioma grade IV had significantly increased ( $p$ value $<0.05$ ) levels of neutrophils, monocytes, NLR, DNLR, and PLR when compared with patients with other grades and healthy controls (-Table 2 ). Patients with glioma grade IV also had significantly lower values ( $p$ value $<0.05$ ) of lymphocytes when compared with healthy controls and other grades of glioma, except for grade III tumors. Patients with glioma grade IV also had significantly lower values ( $p$ value $<0.05$ ) of LMR when compared with other gliomas. 
Table 1 Preoperative characteristics of patients with glioma, meningioma, acoustic neuroma, and healthy controls

\begin{tabular}{|l|l|l|l|l|}
\hline & Healthy control & Acoustic neuroma & Meningioma & Glioma \\
\hline Age & $41(7-82)$ & $49.5(22-78)$ & $54.5(19-86)$ & $42(3-87)$ \\
\hline No of patients & 107 & 36 & 58 & 154 \\
\hline Male & 66 & 14 & 23 & 86 \\
\hline Female & 41 & 22 & 35 & 68 \\
\hline WBC in 109/L & $6.2(3.48-9.29)$ & $5.68(2.05-10.61)^{\mathrm{a}}$ & $5.97(2.93-9.16)$ & $7.27(2.91-17.14)^{\mathrm{a}-\mathrm{c}}$ \\
\hline Neutrophil in 109/L & $3.05(1.67-5.01)$ & $2.81(1.23-5.67)^{\mathrm{a}, \mathrm{c}}$ & $3.20(1.67-5.79)^{\mathrm{b}}$ & $4.31(1.1-13.67)^{\mathrm{a}-\mathrm{c}}$ \\
\hline Lymphocyte in 109/L & $2.29(1.03-3.99)$ & $1.9(0.7-2.98)^{\mathrm{a}}$ & $1.84(0.7-2.93)^{\mathrm{a}}$ & $1.98(0.38-4.5)^{\mathrm{a}, \mathrm{c}}$ \\
\hline Monocytes in 109/L & $0.41(0.23-3.36)$ & $0.45(0.1-0.98)^{\mathrm{a}}$ & $0.40(0.2-1.32)^{\mathrm{b}}$ & $0.47(0.14-2.1)^{\mathrm{a}, \mathrm{c}}$ \\
\hline Eosinophils in 109/L & $0.15(0.01-0.8)$ & $0.24(0.02-0.98)^{\mathrm{a}, \mathrm{c}}$ & $0.13(0.01-0.65)^{\mathrm{b}}$ & $0.34(0.01-0.98)^{\mathrm{a}-\mathrm{c}}$ \\
\hline Platelets in 109/L & $230(2.99-4.91)$ & $225(82-356)$ & $234.5(119-556)^{\mathrm{b}}$ & $222(75-556)^{\mathrm{c}}$ \\
\hline Albumin in g/L & $43(2.99-4.91)$ & $45.45(24-58)^{\mathrm{a}}$ & $43.4(28-58)$ & $41.05(22-53)^{\mathrm{b}}$ \\
\hline NLR & $1.52(0.42-4.36)$ & $1.94(1.75-1.9)^{\mathrm{a}}$ & $1.94(0.84-4.66)^{\mathrm{a}}$ & $2.47(0.44-15.04)^{\mathrm{a}-\mathrm{c}}$ \\
\hline dNLR & $1.06(0.32-2.97)$ & $1.16(1.14-1.5)^{\mathrm{c}}$ & $1.43(0.54-2.87)^{\mathrm{b}}$ & $1.61(0.29-27.93)^{\mathrm{b}, \mathrm{c}}$ \\
\hline ELR & $0.11(0.003-0.67)$ & $0.09(0.002-0.41)$ & $0.08(0.003-0.71)$ & $0.041(0-0.69)^{\mathrm{a}}$ \\
\hline PLR & $111.75(25.6-325.6)$ & $111.39(117.14-119.46)^{\mathrm{c}}$ & $130.8(51.53-615.18)^{\mathrm{a}, \mathrm{b}}$ & $120.66(32.79-510.26)^{\mathrm{a}-\mathrm{c}}$ \\
\hline LMR & $5.6(0.45-13.4)$ & $5.56(7-22.01)^{\mathrm{c}}$ & $4.92(1.68-10.38)^{\mathrm{a}, \mathrm{b}}$ & $4.34(0.3-18.9)^{\mathrm{a}, \mathrm{b}}$ \\
\hline PNI & $61.27(10.96-149.6)$ & $54.72(27.5-56.3)$ & $52.02(34.85-70.8)^{\mathrm{a}}$ & $50.97(25.4-70.45)^{\mathrm{a}, \mathrm{b}}$ \\
\hline
\end{tabular}

Abbreviations: dNLR; derived neutrophil/lymphocyte ratio; ELR, eosinophil/lymphocyte ratio; LMR, lymphocyte/monocyte ratio; NLR, neutrophil/ lymphocyte ratio; PLR, platelet/lymphocyte ratio; PNI, prognostic nutritional index.

a $p<0.05$ compared with healthy controls.

${ }^{\mathrm{b}} p<0.05$ compared with patients with acoustic neuroma.

$c p<0.05$ compared with patients with meningioma

Table 2 Correlations between preoperative inflammatory markers and glioma grade

\begin{tabular}{|c|c|c|c|c|c|}
\hline Marker & Healthy controls & $\begin{array}{l}\text { Glioma grade I } \\
(n=26)\end{array}$ & $\begin{array}{l}\text { Glioma grade II) } \\
(n=38)\end{array}$ & $\begin{array}{l}\text { Glioma grade III } \\
(n=32)\end{array}$ & $\begin{array}{l}\text { Glioma grade IV } \\
(n=58)\end{array}$ \\
\hline WBC in $109 / \mathrm{L}$ & $6.1(3.48-9.29)$ & $6.9(4.74-16.89)^{\mathrm{a}, \mathrm{d}}$ & $6.87(2.91-17.14)^{\mathrm{a}}$ & $7.08(4.38-16.09)^{\mathrm{a}, \mathrm{b}}$ & $8.46(5.91-12.39)^{\mathrm{a}-\mathrm{c}}$ \\
\hline $\begin{array}{l}\text { Neutrophil } \\
\text { in } 109 / \mathrm{L}\end{array}$ & $3.05(1.67-5.01)$ & $3.63(1.97-13.67)^{\mathrm{a}}$ & $3.63(1.1-12.5)^{\mathrm{a}}$ & $4.17(2.56-13.67)^{\mathrm{a}, \mathrm{b}}$ & $6.1(3.67-10.2)^{\mathrm{a}-\mathrm{d}}$ \\
\hline $\begin{array}{l}\text { Lymphocyte } \\
\text { in } 109 / \mathrm{L}\end{array}$ & $2.29(1.03-3.99)$ & $2.43(0.98-4.5)^{\mathrm{a}}$ & $2.03(0.7-4.1)^{\mathrm{a}}$ & $1.98(0.88-2.93)^{\mathrm{a}, \mathrm{b}}$ & $1.615(0.38-4.1)^{\mathrm{a}-\mathrm{c}}$ \\
\hline $\begin{array}{l}\text { Monocytes } \\
\text { in } 109 / \mathrm{L}\end{array}$ & $0.41(0.23-3.36)$ & $0.47(0.21-1.22)^{\mathrm{a}}$ & $0.425(0.2-1.23)$ & $0.45(0.22-0.96)$ & $0.56(0.14-2.1)^{\mathrm{a}-\mathrm{d}}$ \\
\hline $\begin{array}{l}\text { Eosinophils } \\
\text { in } 109 / \mathrm{L}\end{array}$ & $0.15(0.01-0.8)$ & $0.11(0.01-0.98)$ & $0.15(0.01-0.98)$ & $0.125(0.01-0.65)$ & $0.19(0.01-0.98)^{a-d}$ \\
\hline $\begin{array}{l}\text { Platelets } \\
\text { in } 109 / \mathrm{L}\end{array}$ & $230(2.99-4.91)$ & $255.5(82-556)^{\mathrm{a}, \mathrm{d}}$ & $260(75-354)^{\mathrm{a}, \mathrm{d}}$ & $245(82-556)^{\mathrm{ac}}$ & $233.5(113-486)^{b-d}$ \\
\hline Albumin in $\mathrm{g} / \mathrm{L}$ & $43(2.99-4.9)$ & $41.75(32-50)$ & $40.6(24.4-52.6)$ & $41(28-52)$ & $39.45(22-53)^{a}$ \\
\hline NLR & $1.52(0.42-4.36)$ & $1.59(0.48-7.23)^{\mathrm{c,d} d}$ & $1.8(0.44-4.33)^{\mathrm{a}}$ & $2.01(1.28-7.23)^{\mathrm{a}, \mathrm{b}}$ & $3.57(1.38-15.04)^{\mathrm{a}-\mathrm{d}}$ \\
\hline $\mathrm{dNLR}$ & $1.06(0.32-2.97)$ & $1.27(0.38-4.25)$ & $1.38(0.29-16.77)^{\mathrm{a}}$ & $1.59(1.06-5.64)^{\mathrm{a}, \mathrm{b}}$ & $2.185(0.87-27.93)^{\mathrm{a}-\mathrm{d}}$ \\
\hline ELR & $0.11(0.003-0.67)$ & $0.04(0.003-0.67)$ & $0.06(0.002-0.69)^{\mathrm{b}}$ & $0.027(0.003-0.093)$ & $0.03(0.01-0.47)^{c}$ \\
\hline PLR & $111.75(25.6-325.6)$ & $\begin{array}{l}109.56 \\
(44.24-329.91)^{d}\end{array}$ & $\begin{array}{l}103.07 \\
(32.79-282.22)^{d}\end{array}$ & $\begin{array}{l}120.17 \\
(51.53-323.89)^{a-c}\end{array}$ & $\begin{array}{l}143.69 \\
(39.21-510.26)^{a-d}\end{array}$ \\
\hline LMR & $5.6(0.45-13.4)^{d}$ & $5.27(1.55-15)^{d}$ & $5.2(0.8-15.42)^{d}$ & $4.13(1.42-10.10)^{\mathrm{a}-\mathrm{c}}$ & $3.22(0.3-18.9)^{\mathrm{a}-\mathrm{d}}$ \\
\hline $\mathrm{PNI}$ & $61.27(10.96-149.6)^{d}$ & $55.13(44-69.5)^{d}$ & $52.28(27.9-70.45)^{\mathrm{a}}$ & $50.5(34.3-61.35)^{a}$ & $49.92(25.4-68.5)^{\mathrm{a}}$ \\
\hline \multicolumn{2}{|c|}{ IDH mutation positive status } & $0(0 \%)$ & $20(76.92 \%)$ & 19 (59.38\%) & $10(17.24 \%)$ \\
\hline
\end{tabular}

Abbreviations: dNLR; derived neutrophil/lymphocyte ratio; ELR, eosinophil/lymphocyte ratio; IDH, isocitrate dehydrogenase; LMR, lymphocyte/monocyte ratio; NLR, neutrophil/lymphocyte ratio; PLR, platelet/lymphocyte ratio; PNI, prognostic nutritional index.

a $p<0.05$ compared with healthy controls.

${ }^{\mathrm{b}} p<0.05$ compared with grade I glioma patients.

c $p<0.05$ compared with grade II glioma patients.

${ }^{\mathrm{d}} p<0.05$ compared with grade III glioma patients. 
Table 3 Correlation between inflammatory markers and glioma grades

\begin{tabular}{|c|c|c|}
\hline & $R$ & $p$-Value \\
\hline WBC & 0.413 & 0.001 \\
\hline Neutrophils & 0.484 & 0.001 \\
\hline Lymphocytes & -0.24 & 0.001 \\
\hline Monocytes & 0.153 & 0.121 \\
\hline Eosinophils & -0.06 & 0.5601 \\
\hline Albumin & -0.06 & 0.6101 \\
\hline Platelets & 0.11 & 0.076 \\
\hline NLR & 0.431 & 0.001 \\
\hline $\mathrm{dNLR}$ & 0.266 & 0.001 \\
\hline ELR & -0.279 & 0.001 \\
\hline PLR & 0.275 & 0.001 \\
\hline LMR & -0.233 & 0.001 \\
\hline PNI & -0.227 & 0.001 \\
\hline
\end{tabular}

Abbreviations:dNLR; derivedneutrophi//lymphocyteratio;ELR,eosinophil/ lymphocyte ratio; LMR, lymphocyte/monocyte ratio; NLR, neutrophil/ lymphocyte ratio; PLR, platelet/lymphocyte ratio; PNI, prognostic nutritional index.
We found significantly lower values ( $p$ value $<0.05$ ) of platelets in grade IV patients when compared with other grades.

\section{Correlation between Inflammatory Markers and Glioma Grades}

As shown in - Table 3, there was statistically significant positive correlation between tumor grade and white blood cell (WBC) count $(r=0.413, p=0.001)$, neutrophils $(r=0.484$, $p=0.001), \operatorname{NLR}(\mathrm{r}=0.431, p=0.001), \mathrm{dNLR}(\mathrm{r}=0.266, p=0.001)$, and PLR $(r=0.275, p=0.001)$. Statistically significant negative correlation was seen between lymphocytes $(\mathrm{r}=-0.24, p=0.001)$, PNI $(r=-0.227, p=0.001)$, LMR $(r=0.233, p=0.001)$, ELR $(r=-0.279, p=0.001)$ and tumor grade. Eosinophils also showed negative correlation with tumor grade $(r=-0.06)$, however it did not reach the significant value $(p=0.5601)$. NLR showed the most significant positive correlation with glioma grade.

\section{Diagnostic Value of Inflammatory Markers in Glioma} Diagnostic value (AUC) of various inflammatory parameters in diagnosis of glioma from other tumors is shown in - Fig. 1A. Maximum diagnostic efficacy for glioma diagnosis
ROC Curve

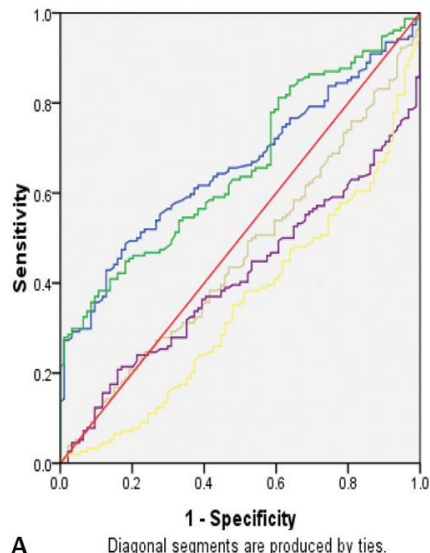

ROC Curve

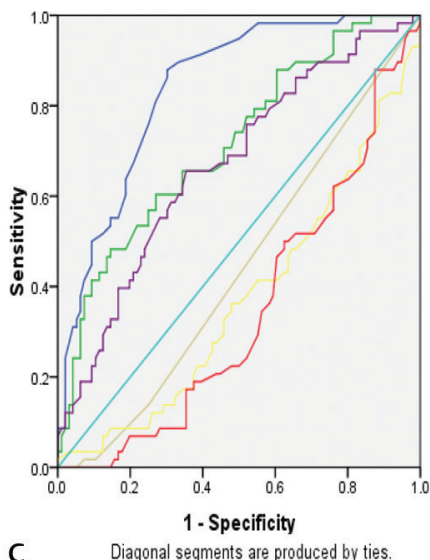

ROC Curve

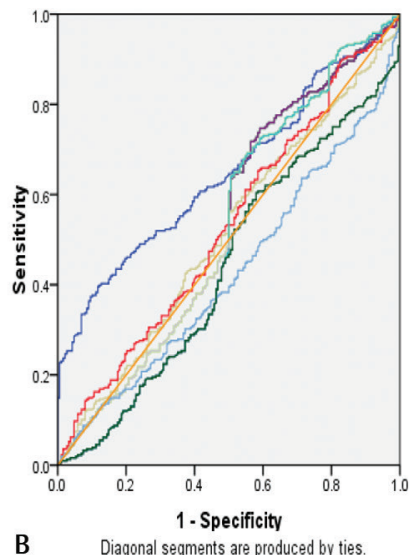

ROC Curve

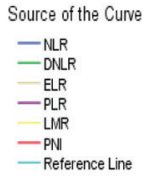

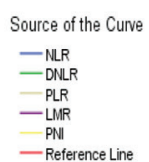

- Reference Line

Fig. 1 Diagnostic value (area under curve $[A \cup C]$ ) of various inflammatory markers and their combinations in diagnosis of glioma versus others (A, B) and glioblastoma multiforme (GBM) versus other grades (C, D). 
was shown by dNLR, with maximum AUC of 0.656639 . The cutoff point for dNLR was 1.26 , with $70.1 \%$ sensitivity and $41.5 \%$ specificity. Among combinations ( Fig. 1B), maximum diagnostic efficacy was shown by NLR + dNLR with AUC of 0.647865 ( - Table 4). The cutoff point for NLR + dNLR was 1.48 , with $70.5 \%$ sensitivity and $42 \%$ specificity.

We also assessed the efficacy of these markers ( - Fig. 1C) and their combinations ( - Fig. 1D) in diagnosis of GBM from other grades (I-III). Among all these markers, NLR (AUC = 0.83926) showed the highest accuracy in diagnosis of GBM and may predict the grade of glioma. The cutoff point for NLR was 2.15 , with $89.7 \%$ sensitivity and $66.7 \%$ specificity. Among combinations, NLR + dNLR showed maximum accuracy (AUC 0.764794). Cutoff point for NLR + dNLR was 1.85, with $78.4 \%$ sensitivity and $59.4 \%$ specificity.

Table 4 Diagnostic value (AUC) of NLR, dNLR, PLR, LMR, PNI, and their combinations

\begin{tabular}{|l|l|l|}
\hline & $\begin{array}{l}\text { GBM vs. } \\
\text { grade I-III }\end{array}$ & $\begin{array}{l}\text { Glioma vs. } \\
\text { others }\end{array}$ \\
\hline Test result variable(s) & Area & Area \\
\hline NLR & 0.83926 & 0.656604 \\
\hline DNLR & 0.714619 & 0.656639 \\
\hline ELR & 0.438757 & 0.428765 \\
\hline PLR & 0.668732 & 0.460037 \\
\hline LMR & 0.372396 & 0.411474 \\
\hline PNI & 0.413214 & 0.449233 \\
\hline NLR-dNLR & 0.764794 & 0.647865 \\
\hline NLR-PLR & 0.62711 & 0.528979 \\
\hline NLR-LMR & 0.57936 & 0.511156 \\
\hline NLR-PNI & 0.546269 & 0.501269 \\
\hline dNLR-PLR & 0.595793 & 0.529143 \\
\hline dNLR-LMR & 0.547346 & 0.529126 \\
\hline dNLR-PNI & 0.515445 & 0.501468 \\
\hline PLR-LMR & 0.511045 & 0.467843 \\
\hline PLR-PNI & 0.49798 & 0.424401 \\
\hline Abreiations: & & \\
\hline
\end{tabular}

Abbreviations: AUC, area under curve; dNLR; derived neutrophil/lymphocyte ratio; ELR, eosinophil/lymphocyte ratio; LMR, lymphocyte/ monocyte ratio; NLR, neutrophil/lymphocyte ratio; PLR, platelet/lymphocyte ratio; PNI, prognostic nutritional index.

\section{Inflammatory Markers and IDH Status}

Our study investigated the difference in values of various inflammatory markers in glioma group to assess their diagnostic value in predicting IDH-wild and IDH-mutated glioma (-Table 5). In our study, there was no IDH mutation in glioma grade I. NLR was significantly different between IDHwild type and IDH-mutated glioma in grade II ( $p$ value $=0.01$; median 1.41 and 1.82 , respectively), grade III ( $p$ value $=0.03$; median 2.01 and 2.41 , respectively) and grade IV glioma ( $p$ value $=0.01$; median 3.47 and 3.91, respectively). - Fig. 2 shows immunoreactivity for IDH 1 , favoring the diagnosis of IDH-mutant glioma.

\section{Discussion}

Chronic inflammation has been accepted as one of the hallmarks for cancer. ${ }^{16}$ Many studies have suggested role of chronic inflammation in cancer pathogenesis. Chronic inflammation results in changes in levels of circulating neutrophils, lymphocytes, monocytes, platelets, eosinophils,

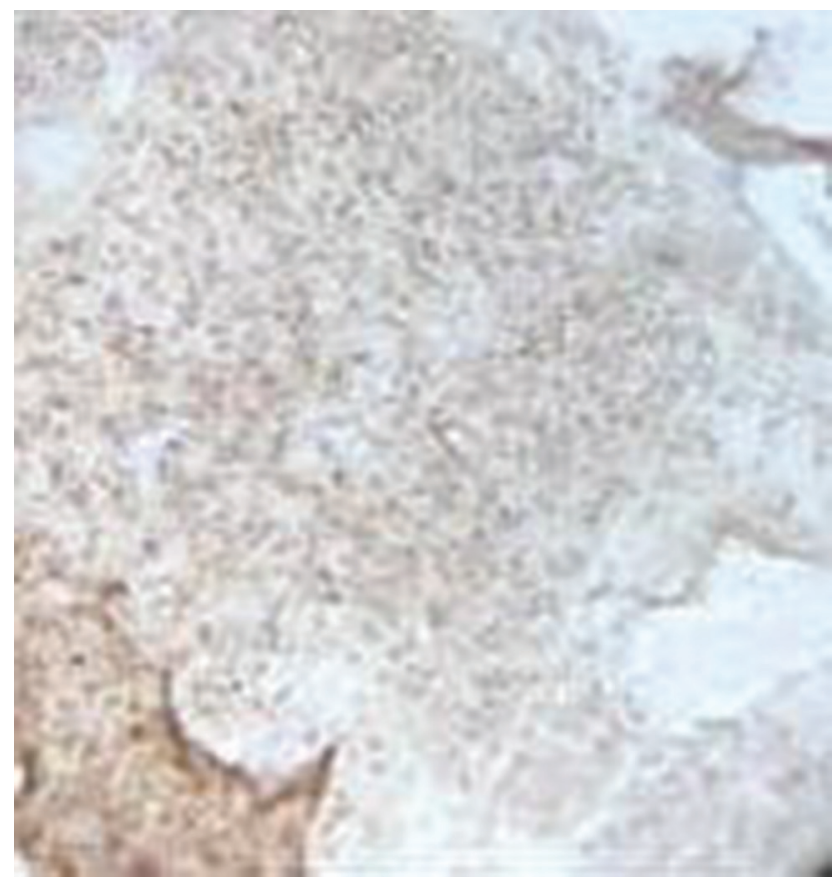

Fig. 2 Isocitrate dehydrogenase (IDH1) immunoreactivity in glioma.

Table 5 Potential influences on ELR, NLR, PLR, LMR, and PNI caused by IDH1 mutation within glioma grade

\begin{tabular}{|l|l|l|l|l|l|l|l|l|l|}
\hline & \multicolumn{3}{|c|}{ Grade II } & \multicolumn{3}{c|}{ Grade III } & \multicolumn{3}{c|}{ Grade IV } \\
\cline { 2 - 11 } & $\begin{array}{l}\text { Wild } \\
\text { type }\end{array}$ & $\begin{array}{l}\text { Mutation } \\
\text { type }\end{array}$ & $p$-Value & $\begin{array}{l}\text { Wild } \\
\text { type }\end{array}$ & $\begin{array}{l}\text { Mutation } \\
\text { type }\end{array}$ & $p$-Value & $\begin{array}{l}\text { Wild } \\
\text { type }\end{array}$ & $\begin{array}{l}\text { Mutation } \\
\text { type }\end{array}$ & $p$-Value \\
\hline Median of ELR & 0.0651 & 0.044 & 0.29 & 0.027 & 0.0297 & 0.81 & 0.0395 & 0.027 & 0.65 \\
\hline Median of NLR & 1.41 & 1.82 & 0.01 & 2.01 & 2.41 & 0.03 & 3.47 & 3.97 & 0.01 \\
\hline Median of PLR & 108.1 & 107.9 & $0 . .41$ & 107.47 & 111.91 & 0.061 & 121.29 & 136.9 & 0.041 \\
\hline Median of LMR & 5.02 & 5.17 & 0.52 & 4.15 & 4.78 & 0.061 & 3.15 & 2.25 & 0.02 \\
\hline Median of PNI & 51.3 & 49.9 & 0.22 & 50.8 & 49.7 & 0.19 & 48.4 & 45.5 & 0.11 \\
\hline
\end{tabular}

Abbreviations: dNLR; derived neutrophil/lymphocyte ratio; ELR, eosinophil/lymphocyte ratio; IDH, isocitrate dehydrogenase; LMR, lymphocyte/monocyte ratio; NLR, neutrophil/lymphocyte ratio; PLR, platelet/lymphocyte ratio; PNI, prognostic nutritional index. 
albumin, and C-reactive protein. ${ }^{17,18}$ High values of preoperative NLR, PLR and low value of preoperative LMR have been suggested as markers for colorectal cancer detection, staging, and treatment response monitoring. They have also been suggested as biomarkers in prostate and lung cancer. Earlier studies have also shown that NLR $>4$ has poor prognosis and shorter survival in GBM. ${ }^{19}$

Studies have recently suggested the role of chronic inflammation in glioma but such studies are very limited. Despite studies suggesting antitumor effect of eosinophils in many solid cancers, eosinophils are scarcely studied in glioma. We aimed to explore the relationship of inflammatory markers in blood to glioma, its grade, and IDH status. We also evaluated the efficacy of these markers in predicting the IDH status of glioma, that is, IDH-wild type versus IDH-mutant type.

The first study on inflammatory markers in glioma by Zadora et $\mathrm{al}^{20}$ concluded that NLR can predict grade of glioma, and a cutoff point of NLR $\geq 2.5$ can predict GBM. Wilson et a ${ }^{21}$ on pediatric glioma reported a significant lower neutrophil count in low-grade glioma (LGG) when compared with high-grade glioma (HGG) and concluded that neutrophil count $\geq 3.36$ can predict death at 2 years after surgery. Although their study reported higher value of NLR in HGG in comparison to LGG but the difference was not significant. A recent study by Wang et al..$^{22}$ in 2018 showed that NLR had the highest diagnostic value for diagnosing GBM from other grades.

None of the above mentioned studies explored the changes in eosinophils in glioma. The study by Costello et $\mathrm{al}^{23}$ showed favorable prognosis when laryngeal carcinomas, urinary bladder carcinoma, and lung cancers were infiltrated by eosinophils, but same eosinophils' infiltration in patients with Hodgkin lymphoma leads to unfavorable prognosis. The mechanism underlying antitumor effect of eosinophils remains unclear. A study suggested that dipeptidyl 4 inhibiter could recruit eosinophils in tumor and also inhibit its growth. ${ }^{24}$ Degranulation phenomenon that leads to release of eosinophil cationic protein (ECP), major basic protein (MBP), eosinophil-derived neurotoxin (EDN), and eosinophil cationic protein (ECP) ${ }^{25}$ is often present with eosinophils in tumor tissue. All these have been suggested to have antitumor effect. Cytokines secreted by eosinophils such as IL-8, TGF- $\alpha$, and IFN- $\gamma$ may have antitumor effect,, ${ }^{26}$ while cytokines like IL- 5 released by eosinophils are linked to worse prognosis. $^{27}$

We found in our study that patients with glioma had significant increase in WBCs, neutrophils, NLR, and dNLR when compared with other tumors and healthy controls. In the study published by Massara et $\mathrm{a}^{28}$ in 2017, neutrophil-induced immunosuppression and angiogenesis were found to promote progression of glioma. The mechanism that results in increase in neutrophils are not clearly defined. Chemotactic factors secretion and generation of reactive oxygen species by the tumor cells may be the underlying mechanism for recruitment of neutrophils in tumor tissue. ${ }^{15}$

Recruitment of neutrophils at tumor site is mediated by CXCL8 secreted by FasL triggering on glioma cells. ${ }^{29}$ Elastase produced by neutrophils at tumor site promotes glioma infiltration and induces proliferation of GBM initiating cells by secreting S100A4. ${ }^{30}$ Neutrophil depletion by monoclonal antibody directed against Ly6G increased survival of mice with glioma. ${ }^{31}$

In our study, we also found significant increase in neutrophils, monocytes, NLR, dNLR, and PLR in GBM when compared with other grades. PLR has been suggested as a marker for prognosis in various cancers. The study by Han et $\mathrm{al}^{32}$ reported that increased PLR was correlated with poor survival. Significantly decreased value of LMR was also observed in patient with grade IV glioma as compared with other grades. Decreased level of LMR has been correlated with poor prognosis in cancers such as urothelial and renal cell carcinoma. ${ }^{33}$ Decreased LMR reflects decrease in lymphocyte count and increase in monocyte count. We also found a negative correlation between lymphocyte, eosinophils, ELR, PNI, and LMR with grade of tumor. Positive correlation was found between TLC, neutrophils, platelets, NLR, dNLR, and PLR. Maximum positive correlation was seen for NLR. In our study, dNLR showed maximum diagnostic efficacy with maximum AUC (0.656639) in diagnosis of glioma from other tumors (cutoff point for dNLR as 1.26 , with $70.1 \%$ sensitivity and $41.5 \%$ specificity). Among the combinations, NLR + dNLR showed the maximum diagnostic efficacy in diagnosis of glioma from other tumors, with cutoff point for NLR + dNLR being 1.85 and sensitivity and specificity $78.4 \%$ and $59.4 \%$, respectively.

Among the various inflammatory markers, maximum positive correlation was found between NLR and PLR. For diagnosis of GBM from other grades, NLR showed the maximum AUC, with cutoff point for NLR being 2.15 and sensitivity and specificity $89.7 \%$ and $66.7 \%$, respectively. Among combinations, NLR + dNLR showed the maximum diagnostic efficacy, with cutoff being 1.85 and sensitivity and specificity $78.4 \%$ and $59.4 \%$, respectively, which is in contrast to earlier studies that have shown NLR + LMR having the highest diagnostic value in predicting grade IV glioma.

We also observed that NLR was the best marker for predicting IDH status of glioma. We did not find IDH mutation in grade I glioma.

Current neuroimaging studies cannot reliably distinguish between different grades of tumor. Even MRI provides $50 \%$ to $80 \%$ diagnostic specificity in diagnosis of GBM from LGG, lymphoma, metastases, and abscess..$^{34}$ Therefore, these inflammatory parameters combined with neuroimaging may be used preoperatively to distinguish glioma, particularly GBM, and may also help in monitoring treatment of glioma and predicting tumor recurrence.

There are certain limitations to the present study. First, the present study consisted of small number of patients and larger studies are therefore required to confirm results. Second, changes in these inflammatory parameters may occur as a nonspecific immune response to glioma or other tumors. Third, data consists of heterogeneous groups, which may decrease the value of results. 


\section{Conclusion}

In this study, we conclude that dNLR has the maximum diagnostic value in diagnosing glioma from other tumors. NLR (AUC $=0.83926)$ showed the highest accuracy for diagnosis of GBM, may predict the grade of glioma, and it also has the maximum diagnostic value in differentiating IDH-wild GBM from IDH-mutant GBM. These peripheral inflammatory parameters may prove to be sensitive and cost-effective markers for glioma diagnosis, predicting grade of glioma, monitoring of treatment response, and in predicting recurrence. Further exploration of these inflammatory changes may lead to opening of new corridors in treatment of glioma. Drugs acting against the protumor effect of neutrophils and promoting the antitumor effect of eosinophils may be the newer drugs in management of advanced glioma.

\section{Conflict of Interest}

None declared.

\section{References}

1 Ostrom QT, Gittleman H, Stetson L, Virk SM, Barnholtz-Sloan JS. Epidemiology of gliomas. Cancer Treat Res 2015; 163:1-14

2 Hochberg FH, Atai NA, Gonda D, et al. Glioma diagnostics and biomarkers: an ongoing challenge in the field of medicine and science. Expert Rev Mol Diagn 2014;14(4):439-452

3 Weller M, van den Bent M, Hopkins K, etal; European Association for Neuro-Oncology (EANO) Task Force on Malignant Glioma. EANO guideline for the diagnosis and treatment of anaplastic gliomas and glioblastoma. Lancet Oncol 2014;15(9): e395-e403

4 Omuro A, DeAngelis LM. Glioblastoma and other malignant gliomas: a clinical review. JAMA 2013;310(17):1842-1850

5 Qu S, Guan J, Liu Y. Identification of microRNAs as novel biomarkers for glioma detection: a meta-analysis based on 11 articles. J Neurol Sci 2015;348(1-2):181-187

6 Hasselbalch HC. Chronic inflammation as a promotor of mutagenesis in essential thrombocythemia, polycythemia vera and myelofibrosis. A human inflammation model for cancer development? Leuk Res 2013;37(2):214-220

7 You J, Zhu GQ Xie L, et al. Preoperative platelet to lymphocyte ratio is a valuable prognostic biomarker in patients with colorectal cancer. Oncotarget 2016;7(18):25516-25527

8 Sato H, Tsubosa Y, Kawano T. Correlation between the pretherapeutic neutrophil to lymphocyte ratio and the pathologic response to neoadjuvant chemotherapy in patients with advanced esophageal cancer. World J Surg 2012;36(3):617-622

9 Xue TC, Zhang L, Xie XY, et al. Prognostic significance of the neutrophil-to-lymphocyte ratio in primary liver cancer: a meta-analysis. PLoS One 2014;9(5):e96072

10 Cedrés S, Torrejon D, Martínez A, et al. Neutrophil to lymphocyte ratio (NLR) as an indicator of poor prognosis in stage IV nonsmall cell lung cancer. Clin Transl Oncol 2012;14(11):864-869

11 Bambury RM, Teo MY, Power DG, et al. The association of pre-treatment neutrophil to lymphocyte ratio with overall survival in patients with glioblastoma multiforme.J Neurooncol 2013;114(1):149-154

12 Han S, Liu Y, Li Q, Li Z, Hou H, Wu A. Pre-treatment neutrophilto-lymphocyte ratio is associated with neutrophil and T-cell infiltration and predicts clinical outcome in patients with glioblastoma. BMC Cancer 2015;15:617

13 Pretlow TP, Keith EF, Cryar AK, et al. Eosinophil infiltration of human colonic carcinomas as a prognostic indicator. Cancer Res 1983;43(6):2997-3000
14 Costello R, O'Callaghan T, Sébahoun G. [Eosinophils and antitumour response]. Rev Med Interne 2005;26(6):479-484

15 Wang, PF, Meng, Z, Song, HW, et al. Preoperative changes in hematological markers and predictors of glioma grade and survival. Front Pharmacol 2018;9:886

16 Hanahan D, Weinberg RA. Hallmarks of cancer: the next generation. Cell 2011;144(5):646-674

17 Deng Q, He B, Liu X, et al. Prognostic value of pre-operative inflammatory response biomarkers in gastric cancer patients and the construction of a predictive model. J Transl Med 2015;13:66

18 Gasparyan AY, Ayvazyan L, Mikhailidis DP, Kitas GD. Mean platelet volume: a link between thrombosis and inflammation? Curr Pharm Des 2011;17(1):47-58

19 McNamara MG, Lwin Z, Jiang H, et al. Factors impacting survival following second surgery in patients with glioblastoma in the temozolomide treatment era, incorporating neutrophil/lymphocyte ratio and time to first progression. J Neurooncol 2014;117(1):147-152

20 Zadora P, Dabrowski W, Czarko K, et al. Preoperative neutrophil-lymphocyte count ratio helps predict the grade of glial tumor - A pilot study. Neurol Neurochir Pol 2015; 49(1):41-44

21 Wilson JRF, Saeed F, Tyagi AK, et al. Pre-operative neutrophil count and neutrophil-lymphocyte count ratio (NLCR) in predicting the histological grade of paediatric brain tumours: a preliminary study. Acta Neurochir (Wien) 2018; 160(4):793-800

22 Wang PF, Meng Z, Song HW, et al. Preoperative changes in hematological markers and predictors of glioma grade and survival. Front Pharmacol 2018;9:886-892

23 Costello R, O'Callaghan T, Sébahoun G. [Eosinophils and antitumour response]. Rev Med Interne 2005;26(6):479-484

24 DPP4 inhibition controls tumor growth via eosinophil recruitment. Cancer Discov 2019;9(4):465

25 Giembycz MA, Lindsay MA. Pharmacology of the eosinophil. Pharmacol Rev 1999;51(2):213-340

26 Sakkal S, Miller S, Apostolopoulos V, Nurgali K. Eosinophils in Cancer:FavourableorUnfavourable?CurrMedChem2016;23(7): 650-666

27 Rosenberg SA. Progress in human tumour immunology and immunotherapy. Nature 2001;411(6835):380-384

28 Massara M, Persico P, Bonavita O, et al. Neutrophils in gliomas. Front Immunol 2017;8:1349

29 Chio CC, Wang YS, Chen YL, Lin SJ, Yang BC. Down-regulation of Fas-L in glioma cells by ribozyme reduces cell apoptosis, tumour-infiltrating cells, and liver damage but accelerates tumour formation in nude mice. $\mathrm{Br} \mathrm{J}$ Cancer 2001; 85(8):1185-1192

30 Liang J, Piao Y, Holmes L, et al. Neutrophils promote the malignant glioma phenotype through S100A4. Clin Cancer Res 2014;20(1):187-198

31 Fujita M, Scheurer ME, Decker SA, et al. Role of type 1 IFNs in antiglioma immunosurveillance-using mouse studies to guide examination of novel prognostic markers in humans. Clin Cancer Res 2010;16(13):3409-3419

32 Han S, Liu Y, Li Q, Li Z, Hou H, Wu A. Pre-treatment neutrophilto-lymphocyte ratio is associated with neutrophil and T-cell infiltration and predicts clinical outcome in patients with glioblastoma. BMC Cancer 2015;15:617

$33 \mathrm{Gu} \mathrm{L}$, Li H, Chen L, et al. Prognostic role of lymphocyte to monocyte ratio for patients with cancer: evidence from a systematic review and meta-analysis. Oncotarget 2016;7(22): 31926-31942

34 Hochberg FH, Atai NA, Gonda D, et al. Glioma diagnostics and biomarkers: an ongoing challenge in the field of medicine and science. Expert Rev Mol Diagn 2014;14(4):439-452 